\title{
Self-assembled Nanostructures: from Nanocrystals to Mesopores and to Nanobelts
}

\author{
Zhong Lin (ZL) Wang*
}

Center for Nanoscience and Nanotechnology, and School of Materials Science and Engineering, Georgia Institute of Technology, Atlanta GA 30332-0245.

* e-mail: zhong.wang@mse.gatech.edu

Size and shape selected nanocrystals behave like molecular matter that can be used as fundamental building blocks for constructing nanocrystal assembled superlattices. The nanocrystals form a new class of materials that have orders in both atomic and nanocrystal length-scales. The nanocrystals are passivated with organic molecules (called thiolates) that not only protect them from coalescence but also act as the molecular bonds for forming the superlattice structure. The interparticle distance is adjustable, possibly resulting in tunable electric, optical and transport properties. This paper reviews our current progress in self-assembly of shape-controlled nanocrystals, photonic crystals and mesoporous materials.

Soft materials made of self-assembled nanocrystals: Self-assembled arrays involve self-organization into monolayers, thin films, and superlattices of size-selected nanocrystals encapsulated in a protective compact organic coating [1]. Nanocrystals are the hard cores that preserve the ordering at the atomic scale; the organic molecules adsorbed on their surfaces serve as the interparticle molecular bonds and as protection for the particles in order to avoid direct core contact with a consequence of coalescing. The interparticle interaction can be changed via control over the length of the molecular chains. Quantum transitions and insulator to conductor transition could be introduced, resulting in tunable electronic, optical and transport properties. We have reported the preparation and structure characterization of threedimensional (3-D) packing of $\mathrm{Au}$ and $\mathrm{Ag}$ nanocrystal super-structures, with an emphasis on the roles played by crystal shapes in forming the nanocrystal superlattice (NCS) [2]. In NCS of Ag nanocrystals with truncated octahedral shape, the type of orientation ordering is determined by the nanocrystal's faceted morphology, as mediated by the interactions of surfactant groups tethered to the facets on neighboring nanocrystals. The particles tend to be packed face-to-face to lower the energy, the surface adsorbed molecules are distributed on the facets of the particles and are self-assembled into bundled and interdigitative molecule bonds [3], which have been found to be extraordinarily strong, stabilizing the NCS to temperatures as high as $500{ }^{\circ} \mathrm{C}$ [4]. The passivation molecules form bundles on the surface, which have been directly observed by energy-filtered TEM [9]. NCS's formed by spherical-like (e.g., icosahedral and decahedral) Ag nanocrystals form hcp superlattices [5]. Defect structures present in NCS's, including: twins, slip planes, multiply-twins and dislocations, have been studied in detail $[1,6]$. The purified $\mathrm{CoO}$ nanocrystals can also form NCS with ordered structure (Figure 3) [7]. CoO particles have a dominant tetrahedral shape of sizes $4 \mathrm{~nm}$. In-situ TEM studies of $\mathrm{CoO}$ and Ag NCS's have proved the structural stability up to temperatures of $\sim 500^{\circ} \mathrm{C}[8]$.

Mesoporous materials as photonic crystals and for low-loss dielectrics and catalysis: There are two main groups of photonic crystals. One is the self-assembly of silica spheres. The other is the ordered porous structure. The ordered hollow structure was made through a template-assisted technique, in which self-assembly of size selective polystyrene spheres creates an ordered template. Metal-organic solution containing the ions for synthesizing the required structure is infiltrated between the spheres. Heating the template at a higher temperature gets rid of the spheres, while the skeleton of the metal oxide still preserves [9]. Ordered self-assembly of hollow structures are potential candidates for low-dielectricconstant materials and catalysis of high surface areas. We have synthesized structures that have ordering and porosity on two length-scales, one is at the scale of hollow spheres created by a template of 
polystyrene (PS) spheres, and the other is the nanocavities created by self-assembled molecular copolymers [10].

Semiconducting oxide nanobelts - a new family of nanomaterials: Ultra-long belt-like, quasi-onedimensional nanostructures (so called nanobelts or nanoribbons) have been successfully synthesized for semiconducting oxides of zinc, tin, indium, cadmium, gallium and lead, by simply evaporating the desired commercial metal oxide powders at high temperatures [11-14]. The as-synthesized oxide nanobelts are pure, structurally uniform, single crystalline and most of them free from defects and dislocations; they have a rectangular-like cross-section with typical widths of 30-300 $\mathrm{nm}$, width-to-thickness ratios of 5-10 and lengths of up to a few millimeters. The belt-like morphology appears to be a unique and common structural characteristic for the family of semiconducting oxides with cations of different valence states and materials of distinct crystallographic structures. The nanobelts are an ideal system for fully understanding dimensionally confined transport phenomena in functional oxides and building functional devices along individual nanobelts [15].

[1] Z.L. Wang, Adv. Mater. 10 (1998) 13.

[2] S.A. Harfenist, Z.L.Wang, et al., J. Phys. Chem. B 100 (1996) 13904.

[3] Z.L. Wang, S.A. Harfenist, et al., J. Phys. Chem. B, 102 (1998) 3068.

[4] S.A. Harfenist and Z.L.Wang, J. Phys. Chem. B, 103 (1999) 4342.

[5] S.A. Harfenist, Z.L.Wang, M.M.Alvarez, I.Vezmar, and R.L.Whetten, Adv. Mater. 9 (1997) 817.

[6] Z.L. Wang, Mater. Charact. 42 (1999) 101.

[7] J.S. Yin and Z.L. Wang, Phys. Rev. Lett. 79 (1997) 2570.

[8] J.S. Yin and Z.L. Wang, J. Phys. Chem. B, 101 (1997) 8979.

[9] J.S. Yin and Z.L. Wang, Adv. Mater. 11 (1999) 469.

[10] J.S. Yin and Z.L. Wang, Appl. Phys. Lett. 74 (1999) 2629.

[11] Z.W. Pan, Z.R. Dai and Z.L. Wang, Science, 291 (2001) 1947.

[12] Z.W. Pan, Z.R. Dai, and Z.L. Wang, Appl. Phys. Letts., 80 (2001) 309.

[13] Z. L. Wang, R. P. Gao, Z. W. Pan and Z. R. Dai, Adv. Eng. Mater., 3, (2001) 657.

[14] Z.R. Dai, Z.W. Pan, Z.L. Wang, Solid State Comm.,118 (2001) 351.

[15] Thanks to the contribution made by my group members: J.S. Yin, S.A. Harfenist, R.L.Whetten, Z.W. Pan, Z.R. Dai, C. Ma and Y. Berta. Thanks for the financial support from NSF grants DMR-9733160.
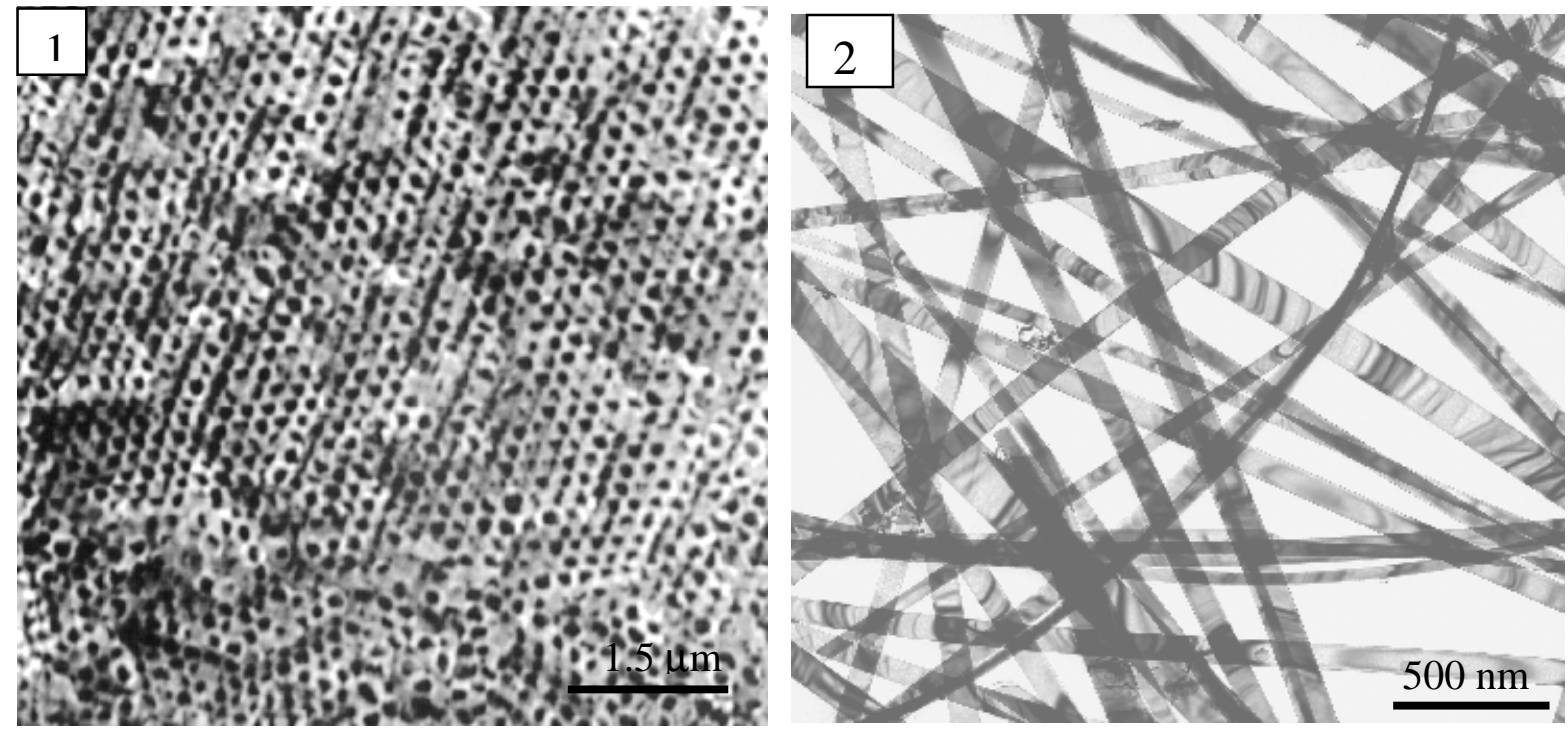

Fig. 1 Scanning electron microscopy images of the porous titania nanostructures.

Fig. 2 Semiconducting $\mathrm{SnO}_{2}$ nanobelts - a new family of nanomaterials. 